\title{
Extracorporeal membrane oxygenation for primary graft dysfunction after heart transplant
}

Scott C. DeRoo, MD, ${ }^{\text {a }}$ Hiroo Takayama, MD, PhD, ${ }^{\mathrm{a}}$ Samantha Nemeth, MA, MPH, ${ }^{\mathrm{a}}$ A. Reshad Garan, MD, ${ }^{\mathrm{b}}$ Paul Kurlansky, MD, ${ }^{\mathrm{a}}$ Susan Restaino, MD, ${ }^{\mathrm{b}}$ Paolo Colombo, MD, Maryjane Farr, MD, ${ }^{b}$ Yoshifumi Naka, $\mathrm{MD}, \mathrm{PhD},{ }^{\mathrm{a}}$ and Koji Takeda, $\mathrm{MD}, \mathrm{PhD}^{\mathrm{a}}$

\section{ABSTRACT}

Objective: Venoarterial extracorporeal membrane oxygenation is a useful treatment for severe primary graft dysfunction after heart transplant. The ideal timing of initiation is unknown.

Methods: We retrospectively reviewed 362 adult heart transplant recipients at our center between January 2011 and December 2017. Thirty-eight patients $(10.5 \%)$ experienced severe primary graft dysfunction treated with venoarterial extracorporeal membrane oxygenation. As our institution adopted a prompt venoarterial extracorporeal membrane oxygenation policy in 2015, patients were stratified into pre-2015 (conservative extracorporeal membrane oxygenation: $\mathrm{n}=18$ ) and post-2015 (prompt extracorporeal membrane oxygenation: $n=20$ ) cohorts Clinical outcomes were compared.

Results: Baseline characteristics were similar (conservative vs prompt) except for age (51.82 vs 59.96 years, $P=.036)$, aspartate transaminase $(32$ vs $21.5 \mathrm{U} / \mathrm{L}$, $P=.038$ ), male donor ( 44.4 vs $80 \%, P=.042$ ), and donor ejection fraction (60 vs $65 \%, P=.047$ ). Median ischemic time was significantly longer in the conservative extracorporeal membrane oxygenation cohort (210 vs 148 minutes, $P=.005)$. Median time to initiation of extracorporeal membrane oxygenation was significantly shorter in the prompt extracorporeal membrane oxygenation cohort (7.26 vs 1.95 hours, $P<.0001)$. There was no difference in intensive care unit stay or major complications. In-hospital mortality improved from $28 \%$ (conservative) to $5 \%$ (prompt, $P=.083$ ). Post-transplant survival at 1 year was $67 \%$ in the conservative extracorporeal membrane oxygenation cohort and $90 \%$ in the prompt extracorporeal membrane oxygenation cohort $(P=.117)$. There was no difference in the Kaplan-Meier survival curves $(P=.071)$, although Cox regression suggested, but certainly did not prove, a 74.6\% lower risk of mortality in the prompt extracorporeal membrane oxygenation group $(P=.094)$.

Conclusions: Prompt venoarterial extracorporeal membrane oxygenation use for primary graft dysfunction after heart transplant results in excellent myocardial recovery and a possible decrease in mortality without increased risk of complications. (J Thorac Cardiovasc Surg 2019;158:1576-84)

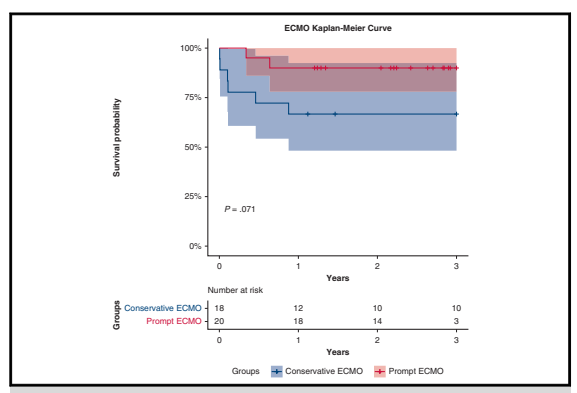

Kaplan-Meier curve comparing survival between the conservative and prompt ECMO cohorts.

\section{Central Message}

Prompt VA-ECMO for severe PGD after heart transplant results in myocardial recovery and a possible decrease in mortality.

\section{Perspective}

Primary graft failure is a devastating complication after heart transplantation. VA-ECMO is useful for the treatment of severe PGD; however, the optimal timing of initiation has not been established. We report that prompt initiation of VA-ECMO in severe PGD allows for excellent myocardial recovery and a possible decrease in mortality without an increased risk of complications.

See Commentaries on pages 1585 and 1587.
From the ${ }^{\mathrm{a}}$ Division of Cardiothoracic Surgery, Department of Surgery, and ${ }^{\mathrm{b}}$ Division of Cardiology, Department of Medicine, Columbia University Medical Center, New York, NY.

Received for publication July 9, 2018; revisions received Jan 13, 2019; accepted for publication Feb 17, 2019; available ahead of print April 1, 2019.

Address for reprints: Koji Takeda, MD, PhD, Columbia University, Department of Cardiothoracic Surgery, 177 Fort Washington Ave, Milstein Hospital Building, 7GN-435, New York, NY 10032 (E-mail: kt2485@cumc.columbia.edu). $0022-5223 / \$ 36.00$

Copyright (c) 2019 by The American Association for Thoracic Surgery https://doi.org/10.1016/j.jtcvs.2019.02.065
Primary graft dysfunction (PGD) is a devastating complication of heart transplantation affecting $2 \%$ to $28 \%$ of all heart transplant recipients. ${ }^{1-4}$ PGD carries a high 30-day

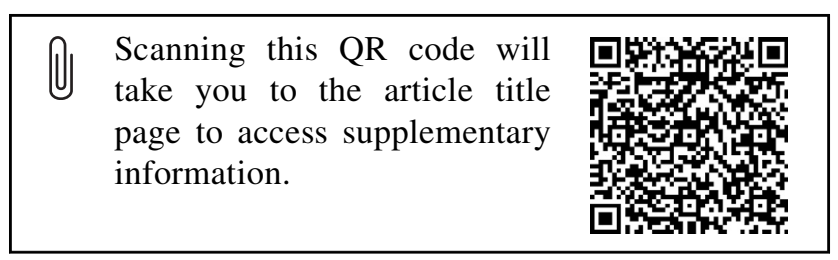




\section{Abbreviations and Acronyms \\ $\mathrm{ECMO}=$ extracorporeal membrane oxygenation \\ MCS = mechanical circulatory support \\ PGD = primary graft dysfunction \\ $\mathrm{VAD}=$ ventricular assist device \\ VA-ECMO $=$ venoarterial extracorporeal membrane oxygenation}

mortality rate of approximately $30 \%$, which is the result of end-organ malperfusion. ${ }^{1}$ Despite multiple advances in the field of transplantation over the past few decades, the factors contributing to PGD and the optimal management of this condition remain incompletely understood. In part, this has been due to the lack of a consensus definition of what constitutes PGD, and subsequently, what treatment modalities provide the greatest benefit. To address these issues, a consensus definition of PGD was proposed by a working group at the International Society for Heart and Lung Transplantation in 2014. ${ }^{1}$ These guidelines have allowed for the standardization and grading of PGD diagnoses, and have provided a more systematic method for assessing the efficacy of PGD treatments.

Currently, several different approaches exist for the management of post-transplant cardiac dysfunction. Mild to moderate PGD can be treated with inotropes or an intraaortic balloon pump; however, severe PGD requires mechanical circulatory support (MCS) to maintain adequate end-organ perfusion. Within the realm of MCS, there are many options for support, including temporary or percutaneous ventricular assist devices (VADs), total artificial heart, and extracorporeal membrane oxygenation (ECMO). Previously, our center demonstrated improved outcomes with the use of ECMO compared with temporary surgically implanted VAD for severe PGD. ${ }^{5}$ On the basis of these results, in 2015 our institution adopted an aggressive ECMO approach for patients with evidence of severe PGD. Specifically, venoarterial extracorporeal membrane oxygenation (VA-ECMO) support is initiated early in our assessment of graft dysfunction in the immediate perioperative period, often during or immediately after weaning from cardiopulmonary bypass. In this study, we evaluated the efficacy of this new approach in the management of PGD.

\section{MATERIALS AND METHODS}

The Columbia University Medical Center Institutional Review Board approved this study with waiver of consent. We retrospectively reviewed 362 adult patients who received orthotopic heart transplantation at Columbia University Medical Center between January 2011 and December 2017. Of these, 38 patients (10.5\%) were placed on ECMO support within 24 hours of transplant because of PGD and were identified and included in this study.

\section{Current Transplant Procedures and Prompt Extracorporeal Membrane Oxygenation Strategy}

All patients underwent cardiac transplantation with bicaval anastomosis. In the prompt ECMO cohort, aortic crossclamp was released after left atrial and aortic anastomoses to shorten the ischemic time. All other anastomoses are performed under beating heart conditions in all cases. Since May 2015, we have adopted an aggressive ECMO approach for patients who display signs of severe isolated right, left, or biventricular failure. Dobutamine, dopamine, milrinone, and epinephrine are used primarily as inotropic agents; norepinephrine and vasopressin are used as vasoactive agents; and epinephrine is considered a mixed inotropic and vasoactive agent. When patients cannot maintain acceptable hemodynamics after weaning from cardiopulmonary bypass (mean arterial pressure $>60 \mathrm{~mm}$ $\mathrm{Hg}$, central venous pressure $<16 \mathrm{~mm} \mathrm{Hg}$, and cardiac index $>2.2 \mathrm{~L} / \mathrm{min} /$ $\mathrm{m}^{2}$ ) despite use of more than 2 high-dose inotropes and 2 high-dose vasopressors, we consider ECMO placement in the operating room at the time of transplant. Before 2015, the decision to initiate ECMO was at the discretion of the attending surgeon. ECMO insertion was often delayed until the patient reached the intensive care unit. Relatively high doses of inotropes and vasopressors were tolerated to maintain a normal mean arterial pressure. Although there was no clear consensus policy on the timing of ECMO initiation in this period, ECMO was typically initiated when indices of perfusion were severely reduced (ie, low mixed venous oxygen saturation, increasing lactate, decreased urine output) in the setting of high doses of vasopressors and inotropes.

Femoral access is our first choice for ECMO cannulation, because moving cannulas away from the chest and allowing for chest closure facilitates patient comfort and early extubation even while remaining on peripheral VA-ECMO. The femoral artery and vein are accessed via open surgical cut-down. A $15 \mathrm{~F}$ or $17 \mathrm{~F}$ arterial and a $21 \mathrm{~F}$ or $23 \mathrm{~F}$ venous cannula (Biomedicus, Medtronic, Minneapolis, Minn) are used in all cases. Partial ECMO flow (3.0 to $4.0 \mathrm{~L} / \mathrm{min}$ ) is maintained in conjunction with inotropes and inhaled nitric oxide to maintain ejection through the aortic valve and pulmonary valve. An $8 \mathrm{~F}$ distal perfusion cannula is routinely inserted into the superficial femoral artery. The sternum is closed after full reversal of heparin with protamine.

\section{Postimplant Extracorporeal Membrane Oxygenation Management}

Anticoagulation with intravenous heparin is initiated at the rate of 300 $\mathrm{U} / \mathrm{h}$ once the chest tube drainage becomes serous. A partial thromboplastin time is measured every 8 hours once heparin is initiated. The partial thromboplastin time goal is slowly up-titrated to 60 to 80 seconds. In an attempt to avoid ventilator-associated pneumonia, we wean ventilator support as soon as possible and extubate patients on ECMO support. ECMO weaning is considered when the patient is improved clinically with evidence of improved graft function, end-organ recovery, and a reduced need for vasoactive medications. Typically, patients are evaluated both by echocardiography and hemodynamic measurements to assess likelihood of ECMO wean. Suitable patients undergo a bedside weaning trial where ECMO flow is briefly decreased to $1 \mathrm{~L} / \mathrm{min}$. Maintenance of satisfactory hemodynamics with acceptable central venous pressure $(<13 \mathrm{~mm} \mathrm{Hg})$ and mean arterial pressure $(>65 \mathrm{~mm} \mathrm{Hg})$ with minimal vasoactive agents, and improvement of graft function on echocardiogram are used to help predict successful weaning from ECMO.

\section{Data Collection and Follow-up}

All clinical data were collected through a review of electronic medical records. Preoperative variables of interest, such as baseline demographics, medical histories, laboratory values, and donor demographics, were collected retrospectively for each patient.

Intraoperative variables included ischemic time, cardiopulmonary bypass time, dose of vasoactive and inotropic medications before ECMO 
initiation, and blood-product use at the time of transplant. The Vasoactive Inotrope Score was calculated on the basis of dose of vasoactive medications as follows: dopamine dose $(\mu \mathrm{g} / \mathrm{kg} / \mathrm{min})+$ dobutamine dose $(\mu \mathrm{g} / \mathrm{kg} /$ $\min )+100 \times$ epinephrine dose $(\mu \mathrm{g} / \mathrm{kg} / \mathrm{min})+10 \times$ milrinone dose $(\mu \mathrm{g} / \mathrm{kg} / \mathrm{min})+10,000 \times$ vasopressin dose (units $/ \mathrm{kg} / \mathrm{min})+100 \times$ norepinephrine dose $(\mu \mathrm{g} / \mathrm{kg} / \mathrm{min}){ }^{6}$ Time from the release of aortic crossclamp to initiation of ECMO was also recorded. Early post-transplant outcomes included mortality, complications during device support, and recovery rate. Echocardiographic data were also recorded at the time of hospital discharge to assess recovery of graft function.

Follow-up examinations were completed on October 17, 2018, and the median follow-up duration was 923 days (interquartile range, 432.231254.5 days). Clinical follow-up was completed in $100 \%$ of patients.

\section{Statistical Analysis}

The "DescTools," "survival," “survminer," “rms," “simPH,” and "ggplot2" packages of $R$ statistical software (version 3.3.3, R Foundation) were used for statistical analysis and all figures. Data are expressed as frequencies and percentages for categorical variables. Continuous variables were checked for normality using the Shapiro-Wilk test. Those found to be normally distributed were expressed as mean \pm standard deviation and compared using the $t$ test. Those variables found to not be normally distributed were expressed as median (interquartile range) and compared using the Mann-Whitney test. Categorical variables were compared using the Fisher exact test. Kaplan-Meier curves were created to represent survival and compared using the log rank test. Because of the low number of mortality events, univariable Cox regression analysis was performed to compare the 2 groups. The hazard ratios were compared using the Wald test.

\section{RESULTS}

\section{Baseline Characteristics at the Time of Heart Transplant}

Patients were stratified into pre-May 2015 (conservative ECMO cohort: $\mathrm{n}=18$ ) and post-May 2015 (prompt ECMO cohort: $\mathrm{n}=20$ ) cohorts. Baseline data and comparisons between the conservative ECMO cohort and the prompt ECMO cohort are summarized in Table 1. Most variables were similar between the 2 groups; however, patients in the conservative ECMO cohort were likely to be younger (mean, 51.82 vs 59.96 years; $P=.036$ ) and have higher aspartate transaminase (median, 32 vs $21.5 \mathrm{U} / \mathrm{L}$; $P=.038$ ) compared with those in the prompt ECMO cohort. Donor characteristics and donor-recipient matching status are also listed in Table 1. The percentage of male donors was higher in the prompt ECMO cohort ( $44 \%$ vs $80 \%$, $P=.042$ ), and donor ejection fraction was higher in the prompt ECMO cohort (median $60 \%$ vs $65 \%, P=.047$ ).

\section{Intraoperative Variables}

Table 2 summarizes the intraoperative variables. Ischemic time was noted to be shorter (median 210 vs 148 minutes, $P=.005$ ); however, the amount of packed red blood cell transfusion (mean 866.67 vs $2410 \mathrm{~mL}$, $P<.0001)$ was greater in the prompt ECMO cohort.

With regard to the timing of ECMO initiation, the prompt ECMO cohort experienced a significantly shorter time to ECMO initiation from crossclamp release (1.95 hours) relative to the conservative ECMO cohort (7.26 hours, $P<.0001)$. Time from the release of aortic crossclamp to initiation of ECMO in each year is shown in Figure E1. The prompt ECMO cohort had a lower dose of epinephrine and milrinone but higher dose of dopamine before ECMO placement. The Vasoactive Inotrope Score was similar between groups. Nineteen patients $(95 \%)$ in the prompt ECMO cohort were placed on peripheral ECMO in the operating room at the time of transplant.

\section{Early and Late Clinical Outcomes}

Early clinical outcomes are displayed in Table 3. Support duration was longer in the prompt ECMO cohort (5.14 vs 3.26 days, $P=.028$ ). Two patients in the conservative ECMO cohort died while on central ECMO support as the result of massive left atrial and left ventricular clot formation and hemorrhage. One patient in the prompt ECMO cohort was managed with central VA-ECMO with a left ventricular vent because of a lack of arterial pulsatility. After 1 day of central ECMO support, this patient was converted to peripheral VA-ECMO. There was no significant difference between groups with regard to major morbidities, including stroke, infection, major bleeding, and renal failure requiring dialysis during ECMO support. Postoperative trends of hepatic and renal markers including aspartate transaminase, alanine transaminase, blood urea nitrogen, and creatinine are shown in Figure E2, A-D. Eight patients $(40 \%)$ in the prompt ECMO cohort were extubated on ECMO support.

With the exception of the 2 patients who died on device support, all patients were able to be weaned from ECMO in both study cohorts. No patients required retransplant in this cohort. Left ventricular ejection fraction was nearly normalized at discharge in both groups.

In the entire cohort, there were 6 in-hospital deaths after weaning ECMO. All died of multiple organ system dysfunction or sepsis despite recovery of graft function. In-hospital mortality decreased from $28 \%$ to $5 \%$ across the study era $(P=.083)$.

One-year survival after transplant was $67 \%$ in the conservative ECMO cohort and $90 \%$ in the prompt ECMO cohort $(P=.117)$, and there was no difference between the 2 cohorts' Kaplan-Meier survival curves $(P=.071)$ (Figure 1). Cox regression showed a $74.6 \%$ reduction in risk of mortality in the prompt ECMO group compared with the conservative group, although with only 8 events the results were not significant (confidence interval, 0.051-1.262; $P=.094$ ) (Figure 2 and Table E1). It should be noted that none of the parameters that differed between groups were found to have an independent association with 3-year survival.

\section{DISCUSSION}

Despite advances in heart transplant management, PGD remains a devastating and poorly understood 
TABLE 1. Baseline characteristics at the time of transplantation

\begin{tabular}{|c|c|c|c|}
\hline & Conservative ECMO $(n=18)$ & Prompt ECMO $(n=20)$ & $P$ value \\
\hline Age, y, mean (SD) & $51.82( \pm 14.21)$ & $59.96( \pm 6.59)$ & .036 \\
\hline Blood type, n (\%) & & & .074 \\
\hline $\mathrm{O}$ & $4(22.22)$ & $12(60.00)$ & \\
\hline A & $9(50.00)$ & $6(30.00)$ & \\
\hline $\mathrm{B}$ & $4(22.22)$ & $2(10.00)$ & \\
\hline $\mathrm{AB}$ & $1(5.56)$ & $0(0.00)$ & \\
\hline BMI, $\mathrm{kg} / \mathrm{m}^{2}$, mean $(\mathrm{SD})$ & $26.60( \pm 4.33)$ & $27.62( \pm 5.28)$ & .518 \\
\hline Race, n (\%) & & & .708 \\
\hline White & $5(27.78)$ & $8(40.00)$ & \\
\hline Black & $9(50.00)$ & $7(35.00)$ & \\
\hline Other & $4(22.22)$ & $5(25.00)$ & \\
\hline Male gender, n (\%) & $16(88.89)$ & $17(85.00)$ & 1.000 \\
\hline Hypertension, n (\%) & $7(38.89)$ & $9(45.00)$ & .752 \\
\hline Diabetes mellitus, n (\%) & $3(16.67)$ & $7(35.00)$ & .278 \\
\hline Hyperlipidemia, n (\%) & $5(27.78)$ & $7(35.00)$ & .734 \\
\hline Cause of heart failure, $\mathrm{n}(\%)$ & & & .883 \\
\hline Ischemic cardiomyopathy & $4(22.22)$ & $6(30.00)$ & \\
\hline Idiopathic dilated cardiomyopathy & $10(55.56)$ & $10(50.00)$ & \\
\hline Congenital & $2(11.11)$ & $1(5.00)$ & \\
\hline Other & $2(11.11)$ & $3(15.00)$ & \\
\hline \multicolumn{4}{|l|}{ Preoperative MCS, n (\%) } \\
\hline Pulsatile VAD & $1(5.56)$ & $0(0.00)$ & .474 \\
\hline Continuous-flow VAD & $12(66.67)$ & $18(90.00)$ & .117 \\
\hline MCS duration, d, median (IQR) & $282.00(214.00-795.00)$ & $463.00(271.00-746.50)$ & .545 \\
\hline Preoperative inotropic support, n (\%) & $3(16.67)$ & $4(20.00)$ & 1.000 \\
\hline Preoperative ventilator support, $\mathrm{n}(\%)$ & $0(0.00)$ & $0(0.00)$ & - \\
\hline Prior sternotomy, n (\%) & $15(83.33)$ & $19(95.00)$ & .328 \\
\hline Repeat transplantation, n (\%) & $0(0)$ & $0(0)$ & - \\
\hline Creatinine, mg/dL, median (IQR) & $1.14(0.85-1.42)$ & $1.42(1.20-1.60)$ & .098 \\
\hline Albumin, g/dL, median (IQR) & $3.90(3.80-4.10)$ & $4.15(3.78-4.43)$ & .328 \\
\hline Total bilirubin, mg/dL, median (IQR) & $0.85(0.50-1.08)$ & $0.60(0.38-0.80)$ & .113 \\
\hline White blood cell count, $\times 1000 / \mathrm{mL}$, median (IQR) & $5.40(4.63-8.13)$ & $6.71(6.16-7.65)$ & .219 \\
\hline Hemoglobin, g/dL, mean (SD) & $11.85( \pm 2.32)$ & $11.18( \pm 2.24)$ & .373 \\
\hline Platelet count, $\times 1000 / \mathrm{mL}$, median $(\mathrm{IQR})$ & $209.50(140.75-290.75)$ & $194.50(161.50-240.25)$ & .619 \\
\hline Hematocrit, $\%$, mean (SD) & $36.50( \pm 6.63)$ & $34.41( \pm 6.63)$ & .337 \\
\hline Sodium, mmol/L, median (IQR) & $137.00(134.50-138.75)$ & $138.50(136.00-140.25)$ & .240 \\
\hline Blood urea nitrogen $\mathrm{mg} / \mathrm{dL}$, median (IQR) & $23.00(14.00-28.50)$ & $25.00(21.75-28.00)$ & .334 \\
\hline Total protein, g/dL, mean (SD) & $6.58( \pm 1.32)$ & $7.00( \pm 0.78)$ & .247 \\
\hline Aspartate transaminase, U/L, median (IQR) & $32.00(24.00-49.00)$ & $21.50(19.00-30.75)$ & .038 \\
\hline Alanine transaminase, U/L, median (IQR) & $24.00(20.25-34.75)$ & $18.00(11.00-24.75)$ & .055 \\
\hline INR & $2.05(1.26-2.40)$ & $2.15(1.30-2.60)$ & .849 \\
\hline Donor age, $y$, median (IQR) & $26.50(21.00-37.00)$ & $33.50(26.00-42.25)$ & .156 \\
\hline Donor blood type, $\mathrm{n}(\%)$ & & & .383 \\
\hline $\mathrm{O}$ & $6(33.33)$ & $11(55.00)$ & \\
\hline A & $9(50.00)$ & $6(30.00)$ & \\
\hline $\mathrm{B}$ & $2(11.11)$ & $3(15.00)$ & \\
\hline
\end{tabular}


TABLE 1. Continued

\begin{tabular}{|c|c|c|c|}
\hline & Conservative ECMO $(n=18)$ & Prompt ECMO $(n=20)$ & $P$ value \\
\hline$\overline{\mathrm{AB}}$ & $1(5.56)$ & $0(0.00)$ & \\
\hline Blood type identical, n (\%) & $16(88.89)$ & $19(95.00)$ & .595 \\
\hline Donor male gender, $\mathrm{n}(\%)$ & $8(44.44)$ & $16(80.00)$ & .042 \\
\hline Gender mismatch, $\mathrm{n}(\%)$ & $10(55.56)$ & $5(25.00)$ & .096 \\
\hline Body weight ratio (donor/recipient), median (IQR) & $0.96(0.85-1.09)$ & $1.01(0.91-1.13)$ & .539 \\
\hline Donor BMI, $\mathrm{kg} / \mathrm{m}^{2}$, median (IQR) & $26.90( \pm 4.90)$ & $26.73( \pm 6.26)$ & .927 \\
\hline Donor ejection fraction, $\%$, median (IQR) & $60.00(57.25-60.00)$ & $65.00(60.00-65.00)$ & .047 \\
\hline
\end{tabular}

Continuous data are shown as the mean \pm SD or the median (IQR). Categorical data are shown as a number (\%). Characteristics were similar except for age, aspartate transaminase, male donor, and donor ejection fraction. ECMO, Extracorporeal membrane oxygenation; SD, standard deviation; BMI, body mass index; $M C S$, mechanical circulatory support; $V A D$, ventricular assist device; $I Q R$, interquartile range.

complication. ${ }^{1,7}$ Although several factors have been implicated in the development of PGD, including donor age, cause of donor brain death, organ preservation technique, ischemia-reperfusion injury, ischemic time, gender mismatch, and the preoperative presence of recipient VAD, there has been little progress in preventing this complication. ${ }^{3,8,9}$ Although prevention of PGD remains the ultimate goal, advances in the treatment and management of this potentially reversible condition may save many lives while we work toward this goal.

MCS is the only effective treatment for severe PGD. ${ }^{2,10-15}$ Prompt implementation of MCS allows for adequate endorgan perfusion while simultaneously allowing the transplanted heart time to recover and regain adequate function while avoiding a period of organ system dysfunction and the long-lasting sequelae that may come of this. Additional advantages of MCS include avoidance of large doses of inotropic or vasoactive agents that may have a potentially harmful effect on the newly transplanted organ. There are several options available to provide temporary hemodynamic support to patients struggling post-transplant. On the basis of our previous study, we began the aggressive use of ECMO as MCS therapy for PGD. Our current study demonstrates that early initiation of ECMO, generally in the same operating room as transplant, results in improved short- and medium-term outcomes with an acceptable

TABLE 2. Intraoperative variables

\begin{tabular}{|c|c|c|c|}
\hline & Conservative ECMO & Prompt ECMO & $P$ value \\
\hline Cardiopulmonary bypass time, min, mean (SD) & $221.11( \pm 69.23)$ & $190.55( \pm 54.46)$ & .143 \\
\hline Ischemic time, min, median (IQR) & $210(158.25-238.00)$ & $148.00(117.75-192.25)$ & .005 \\
\hline \multicolumn{4}{|l|}{ Transfusion, $\mathrm{mL}$} \\
\hline Packed red blood cells, mean (SD) & $866.67( \pm 712.91)$ & $2410( \pm 1688.79)$ & $<.001$ \\
\hline Fresh-frozen plasma, median (IQR) & $1215.00(607.50-2092.50)$ & $1485.00(565.50-3442.50)$ & 639 \\
\hline Platelets, median (IQR) & $490.00(490.00-735.00)$ & $490.00(490.00-980.00)$ & .696 \\
\hline Timing of device implantation after crossclamp release, $h$, median (IQR) & $7.26(3.07-11.70)$ & $1.95(1.32-2.48)$ & $<.001$ \\
\hline ECMO placed in same OR timing, $\mathrm{n}(\%)$ & $9(50.00)$ & $19(95.00)$ & $<.001$ \\
\hline VIS at ECMO insertion & $32.69(13.55)$ & $27.94(6.85)$ & .204 \\
\hline Epinephrine ( $\mu \mathrm{g} / \mathrm{min}$, mean, $\mathrm{SD})$ & $5.22( \pm 3.26)$ & $3.10( \pm 1.86)$ & .022 \\
\hline Dobutamine $(\mu \mathrm{g} / \mathrm{kg} / \mathrm{min}$, median, IQR) & $0.00(0.00-5.00)$ & $0.00(0.00-5.00)$ & .744 \\
\hline Milrinone $(\mu \mathrm{g} / \mathrm{kg} / \mathrm{min}$, median, IQR) & $0.25(0.25-0.38)$ & $0.00(0.00-0.25)$ & .001 \\
\hline Norepinephrine $(\mu \mathrm{g} / \mathrm{min}$, mean, $\mathrm{SD})$ & $12.00( \pm 8.31)$ & $9.75( \pm 5.67)$ & .353 \\
\hline Vasopressin (U/h, median, IQR) & $4.00(4.00-6.00)$ & $4.00(2.00-6.00)$ & .120 \\
\hline Dopamine $(\mu \mathrm{g} / \mathrm{kg} / \mathrm{min}$, median, IQR) & $0.00(0.00-0.00)$ & $5.00(4.50-5.00)$ & $<.001$ \\
\hline Type of ECMO, n (\%) & & & .083 \\
\hline Central & $5(27.78)$ & $1(5.00)$ & \\
\hline Peripheral & $13(72.22)$ & $19(95.00)$ & \\
\hline
\end{tabular}

Continuous data are shown as the mean \pm SD or the median (IQR). Categorical data are shown as a number (\%). Median ischemic time was significantly longer in the conservative ECMO cohort, and median time to initiation of ECMO was significantly shorter in the prompt ECMO cohort. ECMO, Extracorporeal membrane oxygenation; $S D$, standard deviation; $I Q R$, interquartile range; $O R$, operating room; VIS, vasoactive inotrope score. 
TABLE 3. Early outcomes

\begin{tabular}{|c|c|c|c|}
\hline & Conservative ECMO & Prompt ECMO & $P$ value \\
\hline Support duration, $\mathrm{d}$, median (IQR) & $3.26(2.48-5.04)$ & $5.14(3.73-7.94)$ & .028 \\
\hline Death on device support, $\mathrm{n}(\%)$ & $2(11.11)$ & $0(0.00)$ & .218 \\
\hline Recovery (weaned off device), n (\%) & $16(88.89)$ & $20(100.00)$ & .218 \\
\hline ICU length of stay, $d$, median (IQR) & $15.00(9.00-30.25)$ & $13.00(8.75-28.00)$ & .884 \\
\hline Retransplant, n (\%) & $0(0.00)$ & $0(0.00)$ & - \\
\hline Extubation on ECMO, n (\%) & $0(0.00)$ & $8(40.00)$ & .003 \\
\hline LVEF at time of hospital discharge, $\%$, median (IQR) & $60.00(60.00-61.25)$ & $60.00(58.75-65.00)$ & .852 \\
\hline \multicolumn{4}{|l|}{ Major morbidity during support, $\mathrm{n}(\%)$} \\
\hline Ventilator-associated pneumonia & $7(38.89)$ & $8(40.00)$ & 1.000 \\
\hline Stroke & $0(0.00)$ & $0(0.00)$ & - \\
\hline Infection & $4(22.22)$ & $4(20.00)$ & 1.000 \\
\hline Major bleeding & $7(38.89)$ & $5(25.00)$ & .489 \\
\hline Vascular injury or ischemic leg & $0(0.00)$ & $0(0.00)$ & - \\
\hline Renal failure requiring dialysis & $1(5.56)$ & $1(5.00)$ & 1.000 \\
\hline \multicolumn{4}{|l|}{ Mortality, n (\%) } \\
\hline $30-d$ & $2(11.11)$ & $0(0.00)$ & .218 \\
\hline In-hospital & $5(27.78)$ & $1(5.00)$ & .083 \\
\hline 1-y mortality & $6(33.33)$ & $2(10.00)$ & .117 \\
\hline
\end{tabular}

Continuous data are shown as the mean \pm SD or the median (IQR). Categorical data are shown as a number ( $\%)$. There was no difference between cohorts in intensive care unit stay or major complications. In-hospital mortality improved from $28 \%$ (conservative) to $5 \%$ (prompt, $P=.083$ ). Post-transplant survival at 1 year was $67 \%$ in the conservative ECMO cohort and $90 \%$ in the prompt ECMO cohort $(P=.117)$. There was no statistically significant difference in outcomes between the 2 groups. ECMO, Extracorporeal membrane oxygenation; $I Q R$, interquartile range; $I C U$, intensive care unit; $L V E F$, left ventricular ejection fraction.

side-effect and complication profile. Our in-hospital mortality of $5 \%$ and 1 -year survival of $90 \%$ in the post- 2015 cohort compares favorably to previously published studies and may represent an incremental improvement in the management of PGD at our institution. ${ }^{2,5,11,15-18}$ Although our sample size is small, we believe that the difference in outcomes likely reflects our prompt, aggressive ECMO approach for this cohort of patients.

Of note, although the timing of ECMO initiation between the conservative and prompt ECMO cohorts differed by approximately 5 hours, it should be noted that both groups underwent relatively early placement of ECMO when compared with several other studies. ${ }^{2,5,18}$ Previous data have demonstrated that delayed ECMO initiation beyond 24 hours in heart transplant recipients with poor hemodynamics results in poor outcomes. This study further refines the concept of prompt ECMO initiation to within the first few hours of surgery, and ideally before leaving the operating room at the time of transplant. Given that PGD starts immediately after reperfusion, we believe that this early, aggressive strategy makes the most sense physiologically and prevents the serious consequences of several hours of poor or impaired endorgan perfusion. Additionally, the prompt use of ECMO rests the newly transplanted heart without exposing it to a period of significant hemodynamic strain and a selfperpetuating cascade of injurious and inflammatory mediators.
The decision to institute ECMO post-transplant is often difficult given the high reported incidence of associated complications. One potential criticism of our approach is that perhaps it is too aggressive and results in the overuse of ECMO. However, advances in the management of patients on ECMO and changes in the physical components of the ECMO circuit have helped reduce the complication rate of ECMO at our institution and have allowed us to use an aggressive ECMO approach while minimizing potential negative side effects. Potentially devastating complications such as stroke and limb ischemia have been reduced to near-zero with tight monitoring and adherence to an anticoagulation regimen, and the routine use of small-caliber perfusion cannula and placement of a distal perfusion cannula for all patients have further improved outcomes. ${ }^{19} \mathrm{We}$ have begun using near-infrared spectroscopy to monitor leg saturations in all patients on ECMO and have been able to more easily identify those patients experiencing reduced or absent flow to a distal extremity. ${ }^{20}$ Taken together, these interventions help minimize the complications and risk associated with ECMO and improve the risk profile of this therapy.

Our goal in patients with severe PGD is to provide partial hemodynamic support with peripheral ECMO cannulation. Typically, we prefer to cannulate the femoral vessels via surgical cut-down. This allows us to close the chest without the need to reopen for decannulation and simplifies the decannulation process. Furthermore, patients can be extubated 


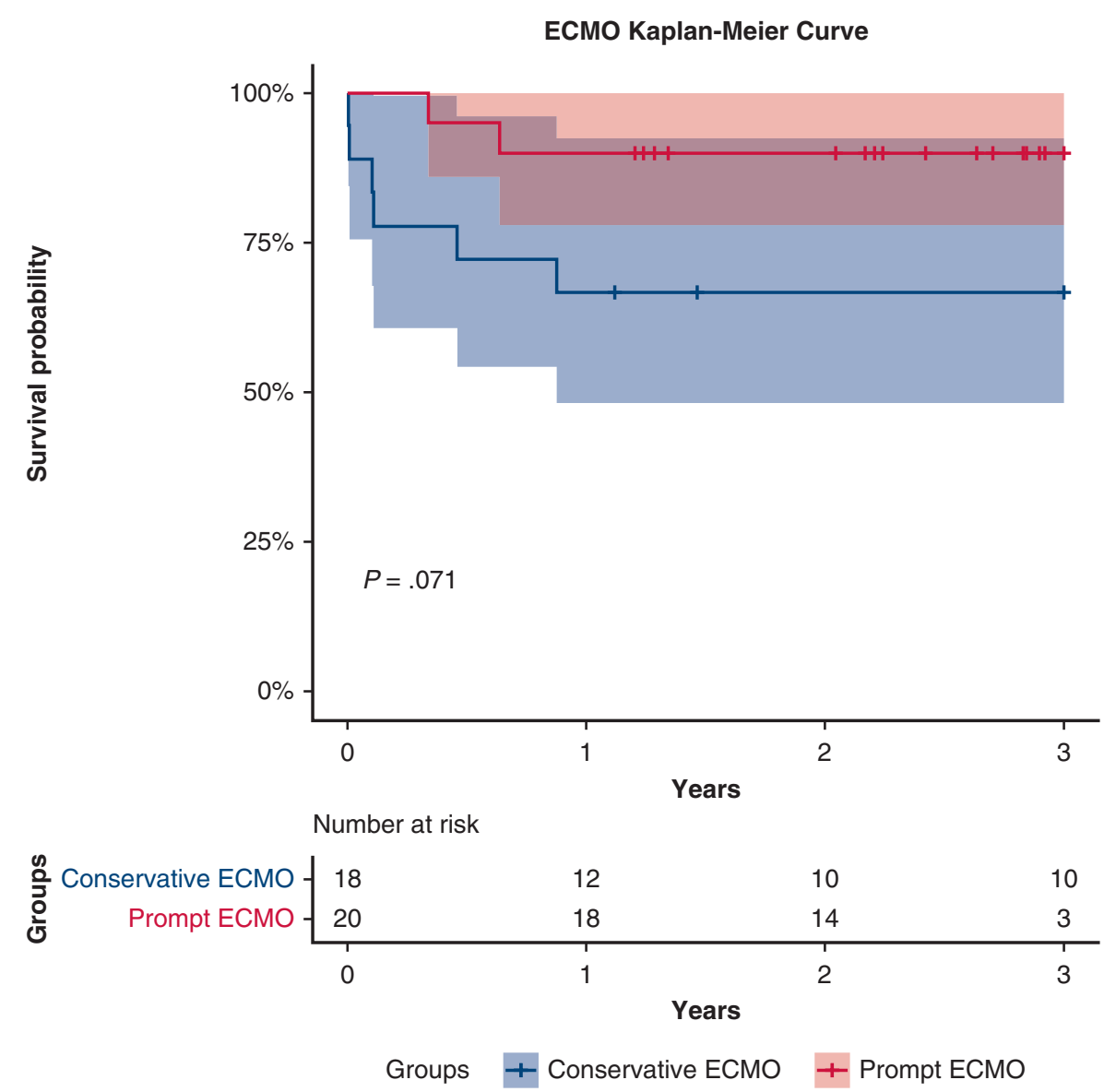

FIGURE 1. Kaplan-Meier curve comparing survival between the conservative ECMO cohort and the prompt ECMO cohort. There was no difference in survival between the 2 groups. ECMO, Extracorporeal membrane oxygenation.

on ECMO support. We think these are beneficial to avoid infectious complications such as sternal infection and pneumonia associated with central cannulation. During the time that patients are maintained on partial ECMO support, our management protocol entails titrating the ECMO flow, inotropic support, and volume to provide the necessary total cardiac output while still allowing for both right and left ventricular ejection as assessed by pulsatility on the Swan-Ganz catheter and arterial line, respectively. This is done to prevent stasis in the intracardiac chambers, which was seen in the conservative ECMO cohort. Admittedly, some patients demonstrate such poor initial graft function that this is not possible; however, we have found that this is extremely rare and allograft function generally starts improving within the first 24 to 48 hours of partial ECMO support. Given that the complications of ECMO therapy increase over time, it is important to note that the average duration of ECMO support in this study was 5 days in the post-2015 cohorts. This demonstrates that the majority of patients with PGD attain full cardiac recovery within several days of MCS support if appropriately managed.
In the coming months in 2018, the New Heart Allocation Policy will prioritize patients who require ECMO as a bridge to heart transplantation (optn.hrsa.transplant.gov, referenced June 26, 2018). Historically, many patients with PGD were treated with maximal medical or mechanical support as available, urgently relisted, and underwent transplantation. In the contemporary era, a patient who has severe PGD requiring ECMO could be urgently listed and undergo retransplantation under the new policy. Our data strongly support the use of prompt ECMO for PGD, but with the goal of recovery and wean rather than urgent retransplant. We believe this strategy is prudent and plausible, and demonstrates good organ stewardship in addition to providing superior outcomes for patients versus urgent retransplantation.

\section{Study Limitations}

There are several limitations to this study. First, this is a retrospective analysis of a single-center experience. Second, the number of patients and events in each group is low, thus limiting its statistical power. Third, because of 


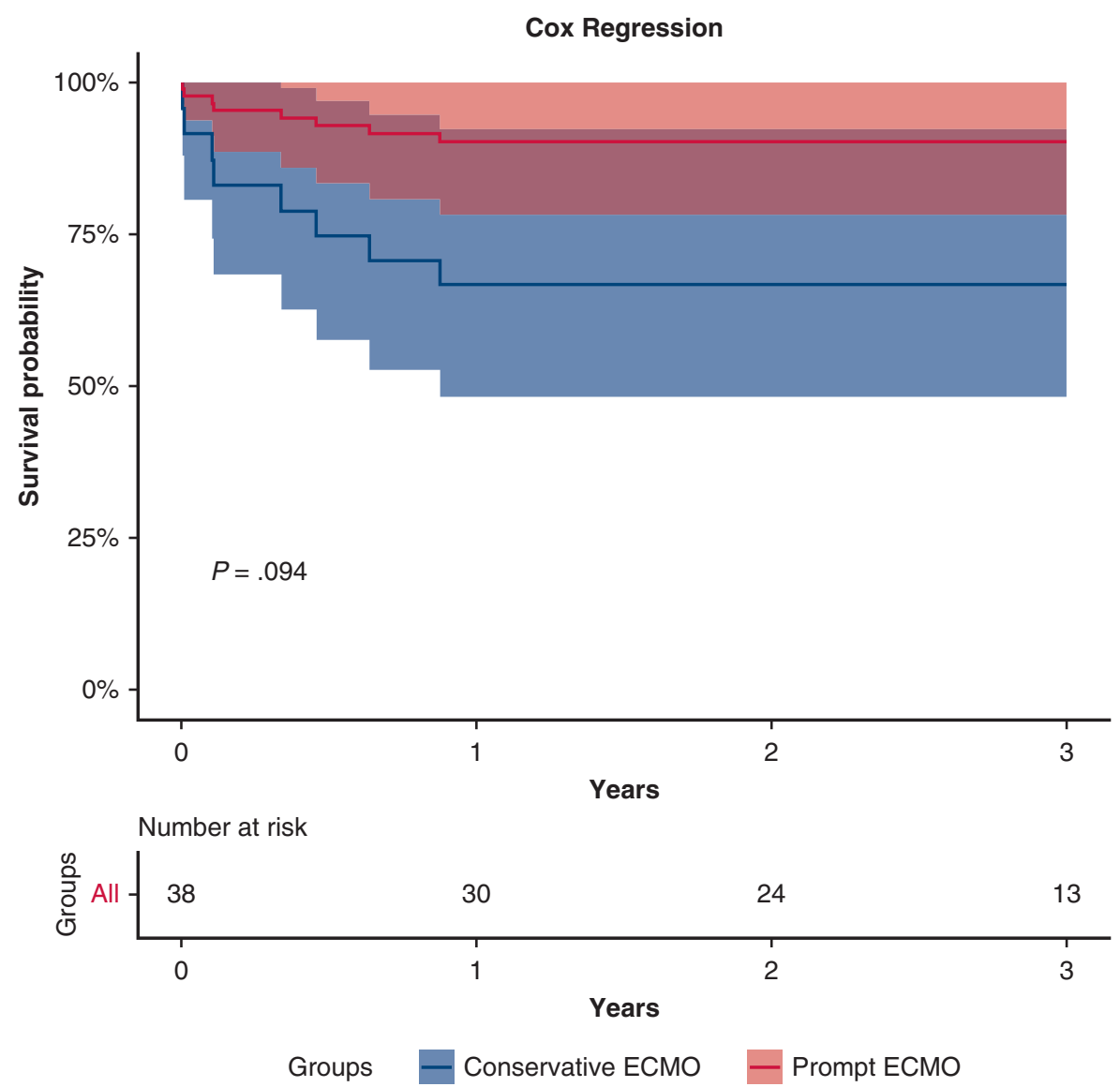

FIGURE 2. Cox proportional hazard model comparing survival between the conservative ECMO cohort and the prompt ECMO cohort. Cox regression suggested but did not prove a $74.6 \%$ lower risk of mortality in the prompt ECMO group $(P=.094)$. ECMO, Extracorporeal membrane oxygenation.

the nature of single-center studies, the outcomes described are based on our practice in terms of patient selection, surgery, and management. Therefore, our findings may not be applicable to other centers. Further, multicenter studies including larger numbers of patients are desirable. Finally, this analysis between groups was a historical comparison as our strategy has changed over time. Although both cohorts were largely comparable with regard to preoperative variables, there are a few differences, and none of these appeared to have any impact on survival. Moreover, especially in the conservative ECMO cohort, the decision to initiate ECMO was at the discretion of the surgeon. Because of the retrospective nature of the study, we cannot overcome this limitation.

\section{CONCLUSIONS}

Our study demonstrates that early and aggressive initiation of ECMO for patients with severe PGD results in excellent allograft recovery and suggests a decrease in mortality. Future studies with larger sample sizes will be needed to confirm the validity of this approach. In the modern era,
ECMO has an acceptably low incidence of complications. When used as a bridge to recovery after heart transplantation with severe PGD, ECMO offers the possibility of an excellent long-term outcome in the face of a historically challenging disease.

\section{Conflict of Interest Statement}

Y.N. received consulting fees from Abbott Laboratories. P.C. received research funding, and serves as a consultant with no honoraria, for Abbott Laboratories. All other authors have nothing to disclose with regard to commercial support.

\section{References}

1. Kobashigawa J, Zuckermann A, Macdonald P, Leprince P, Esmailian F, Luu M, et al. Report from a consensus conference on primary graft dysfunction after cardiac transplantation. J Heart Lung Transplant. 2014;33:327-40.

2. Lima EB, Cunha CR, Barzilai VS, Ulhoa MB, Barros MR, Moraes CS, et al. Experience of ECMO in primary graft dysfunction after orthotopic heart transplantation. Arq Bras Cardiol. 2015;105:285-91.

3. Russo MJ, Iribarne A, Hong KN, Ramlawi B, Chen JM, Takayama H, et al. Factors associated with primary graft failure after heart transplantation. Transplantation. 2010;90:444-50. 
4. Iyer A, Kumarasinghe G, Hicks M, Watson A, Gao L, Doyle A, et al. Primary graft failure after heart transplantation. J Transplant. 2011;2011:175768.

5. Takeda K, Li B, Garan AR, Topkara VK, Han J, Colombo PC, et al. Improved outcomes from extracorporeal membrane oxygenation versus ventricular assist device temporary support of primary graft dysfunction in heart transplant. $J$ Heart Lung Transplant. 2017;36:650-6.

6. Gaies MG, Gurney JG, Yen AH, Napoli ML, Gajarski RJ, Ohye RG, et al. Vasoactive-inotropic score as a predictor of morbidity and mortality in infants after cardiopulmonary bypass. Pediatr Crit Care Med. 2010;11:234-8.

7. Stehlik J, Edwards LB, Rowe A, Philibin K, Williamson J, Kirklin JK, et al. ISHLT International Registry for Heart and Lung Transplantation-three decades of scientific contributions. Transplant Rev (Orlando). 2013;27:38-42.

8. Segovia J, Pulpon LA, Sanmartin M, Tejero C, Serrano S, Burgos R, et al. Primary graft failure in heart transplantation: a multivariate analysis. Transplant Proc. 1998:30:1932.

9. Young JB, Hauptman PJ, Naftel DC, Ewald G, Aaronson K, Dec GW, et al. Determinants of early graft failure following cardiac transplantation, a 10-year, multiinstitutional, multivariable analysis. J Heart Lung Transplant. 2001;20:212.

10. Kavarana MN, Sinha P, Naka Y, Oz MC, Edwards NM. Mechanical support for the failing cardiac allograft: a single-center experience. J Heart Lung Transplant. 2003;22:542-7.

11. Kittleson MM, Patel JK, Moriguchi JD, Kawano M, Davis S, Hage A, et al. Heart transplant recipients supported with extracorporeal membrane oxygenation: outcomes from a single-center experience. J Heart Lung Transplant. 2011;30:1250-6.

12. Listijono DR, Watson A, Pye R, Keogh AM, Kotlyar E, Spratt P, et al. Usefulness of extracorporeal membrane oxygenation for early cardiac allograft dysfunction. J Heart Lung Transplant. 2011;30:783-9.

13. Mihaljevic T, Jarrett CM, Gonzalez-Stawinski G, Smedira NG, Nowicki ER, Thuita L, et al. Mechanical circulatory support after heart transplantation. Eur J Cardiothorac Surg. 2012;41:200-6.
14. Su JA, Kelly RB, Grogan T, Elashoff D, Alejos JC. Extracorporeal membrane oxygenation support after pediatric orthotopic heart transplantation. Pediatr Transplant. 2015;19:68-75.

15. Taghavi S, Ankersmit HJ, Wieselthaler G, Gorlitzer M, Rajek A, Wolner E, et al. Extracorporeal membrane oxygenation for graft failure after heart transplantation: recent Vienna experience. J Thorac Cardiovasc Surg. 2001; 122:819-20.

16. D'Alessandro C, Aubert S, Golmard JL, Praschker BL, Luyt CE, Pavie A, et al. Extra-corporeal membrane oxygenation temporary support for early graft failure after cardiac transplantation. Eur J Cardiothorac Surg. 2010; 37:343-9.

17. Kaushal S, Matthews KL, Garcia X, Wehman B, Riddle E, Ying Z, et al. A multicenter study of primary graft failure after infant heart transplantation: impact of extracorporeal membrane oxygenation on outcomes. Pediatr Transplant. 2014;18:72-8.

18. Marasco SF, Vale M, Pellegrino V, Preovolos A, Leet A, Kras A, et al. Extracorporeal membrane oxygenation in primary graft failure after heart transplantation. Ann Thorac Surg. 2010;90:1541-6.

19. Takayama H, Landes E, Truby L, Fujita K, Kirtane AJ, Mongero L, et al. Feasibility of smaller arterial cannulas in venoarterial extracorporeal membrane oxygenation. J Thorac Cardiovasc Surg. 2015;149:1428-33.

20. Patton-Rivera K, Beck J, Fung K, Chan C, Beck M, Takayama H, et al. Using near-infrared reflectance spectroscopy (NIRS) to assess distal-limb perfusion on venoarterial (V-A) extracorporeal membrane oxygenation (ECMO) patients with femoral cannulation. Perfusion. 2018;33:618-23.

Key Words: extracorporeal membrane oxygenation, ECMO, heart transplant, primary graft failure

Readers who found these articles interesting may also like to read the following papers found in recent and future issues of our sister publications, Seminars in Thoracic and Cardiovascular Surgery and Operative Techniques in Thoracic and Cardiovascular Surgery!

\section{Adult: Mechanical Circulatory Support}

STCVS Invited: State of the Art: Interhospital ECMO Transport: Regional Focus. Bonadonna D, Barac YD, Ranney DN, et al. Semin Thorac Cardiovasc Surg. 2019;31(3):327-334. 


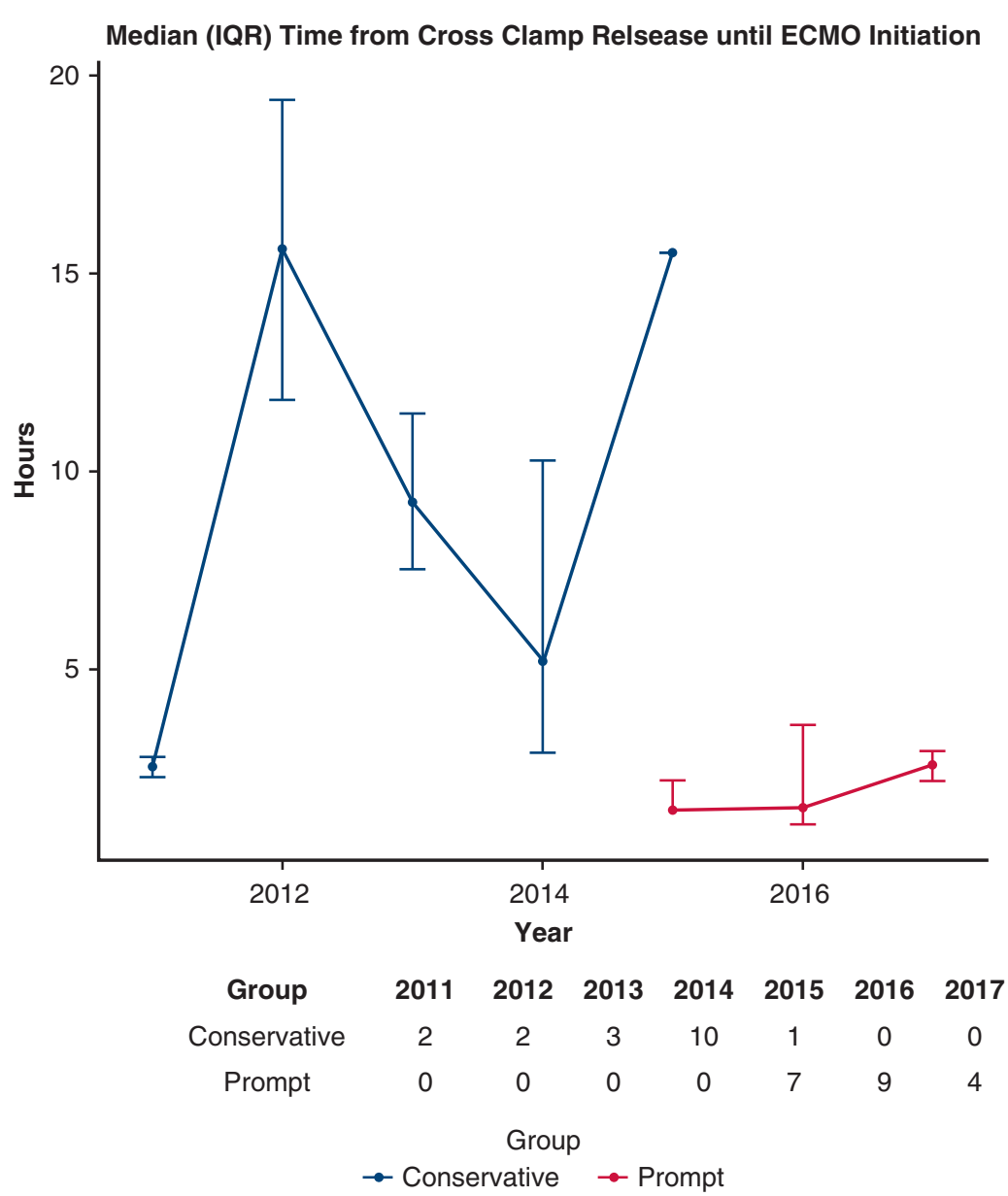

FIGURE E1. Time to initiation of ECMO from release of aortic crossclamp by year. $I Q R$, Interquartile range; ECMO, extracorporeal membrane oxygenation. 

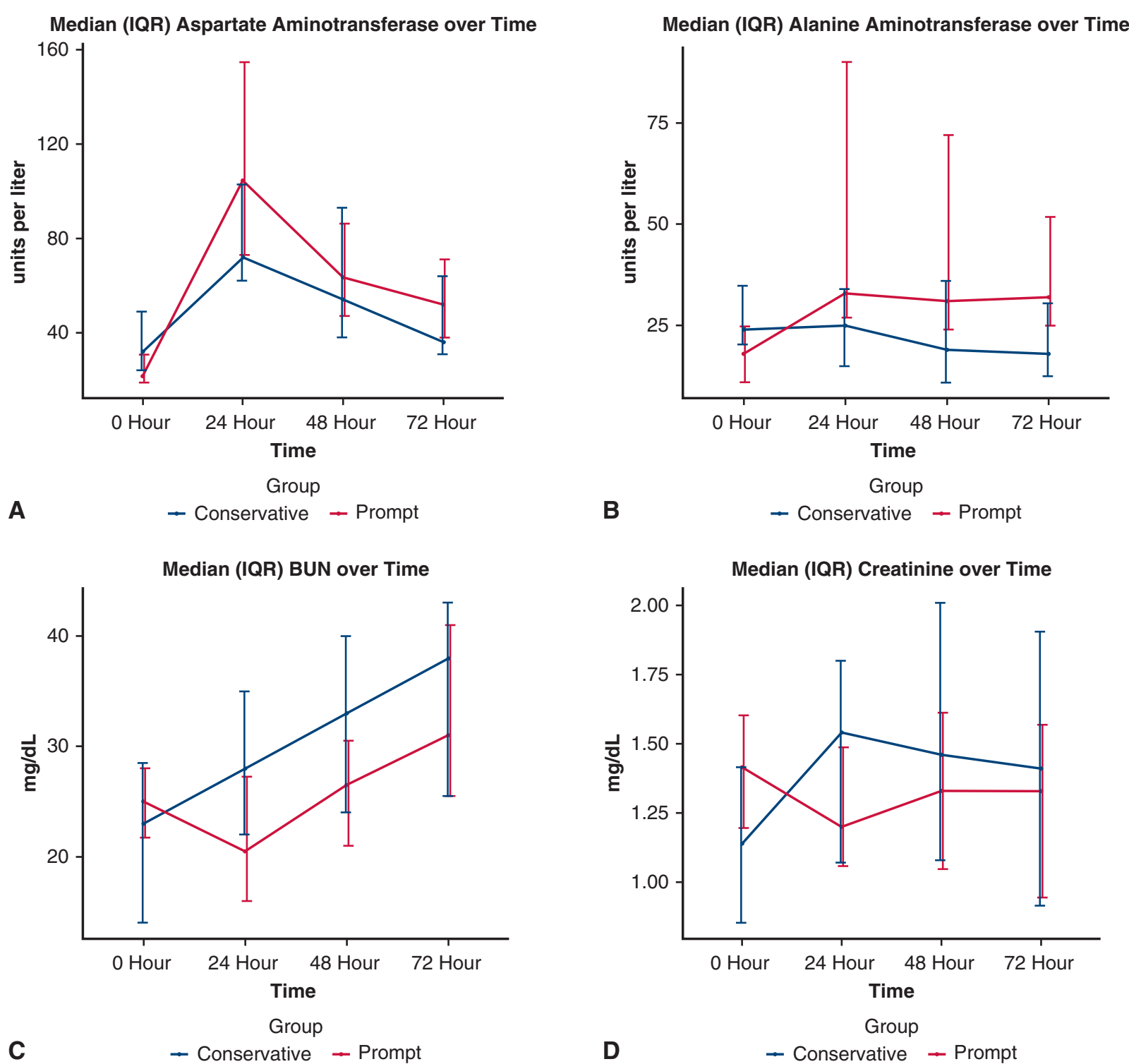

FIGURE E2. Trend of hepatic and renal function markers over time. A, Aspartate aminotransferase. B, Alanine aminotransferase. C, Blood urea nitrogen. $\mathrm{D}$, Creatinine. $I Q R$, Interquartile range; $B U N$, blood urea nitrogen. 
TABLE E1. Univariate Cox regression

\begin{tabular}{|c|c|c|c|c|c|}
\hline Field & HR & Lower 95 CI & Upper 95 CI & $P$ value & $\begin{array}{l}\text { Proportional hazard } \\
\text { assumption held }\end{array}$ \\
\hline Group & 0.254 & 0.051 & 1.262 & .094 & 0.331 \\
\hline Age & 1.003 & 0.944 & 1.066 & .916 & 0.623 \\
\hline Mechanical circulator support duration & 1.001 & 0.999 & 1.003 & .25 & 0.917 \\
\hline Donor gender $(\mathrm{M}=1)$ & 1.024 & 0.245 & 4.285 & .975 & 0.266 \\
\hline Ischemic time & 1.015 & 0.996 & 1.035 & .132 & 0.678 \\
\hline Transfusion red blood cells & 1 & 0.9996 & 1 & .854 & 0.719 \\
\hline Support duration & 1.114 & 0.954 & 1.302 & .173 & 0.819 \\
\hline Extubation on ECMO & 0.481 & 0.059 & 3.912 & .494 & 0.264 \\
\hline Creatinine & 0.691 & 0.118 & 4.069 & .683 & 0.915 \\
\hline Donor ejection fraction & 0.891 & 0.782 & 1.012 & .077 & 0.339 \\
\hline
\end{tabular}

$H R$, Hazard ratio; $C I$, confidence interval; ECMO, extracorporeal membrane oxygenation. *Only 8 events in the dataset Schoenfeld Residuals Test. 